\title{
Video-Rate Lissajous-Scan Atomic Force Microscopy
}

\author{
Yuen Kuan Yong, Member, IEEE, Ali Bazaei, Member, IEEE, and S. O. Reza Moheimani, Fellow, IEEE
}

\begin{abstract}
Raster scanning is common in atomic force microscopy (AFM). The nonsmooth raster waveform contains highfrequency content that can excite mechanical resonances of an AFM nanopositioner during a fast scan, causing severe distortions in the resulting image. The mainstream approach to avoid scan-induced vibrations in video-rate AFM is to employ a highbandwidth nanopositioner with the first lateral resonance frequency above $20 \mathrm{kHz}$. In this paper, video-rate scanning on a nanopositioner with $11.3-\mathrm{kHz}$ resonance frequency is reported using a smooth Lissajous scan pattern. The Lissajous trajectory is constructed by tracking two sinusoidal waveforms on the lateral axes of the nanopositioner. By combining an analog integral resonant controller (IRC) with an internal model controller, 1- and $2-\mathrm{kHz}$ single tone set-points were successfully tracked. Highquality time lapsed AFM images of a calibration grating recorded at 9 and 18 frames/s without noticeable image distortions are reported.
\end{abstract}

Index Terms-Atomic force microscopy, flexure-based, integral resonant control, internal model control, Lissajous-scan, nonraster scanning, video-rate.

\section{INTRODUCTION}

$\mathbf{T}$ HE invention of scanning tunneling microscopy (STM) in early 1980s facilitated imaging of conducting sample surfaces on atomic scale [1]. The need for imaging nonconducting surfaces, at resolutions beyond the reach of optical microscopy, led to the invention of atomic force microscopy (AFM) later in 1986 [2]. Since its debut, the AFM has become one of the most versatile instruments for studying objects on the nanoscale. It has been used to interrogate, manipulate, and image a variety of samples in vacuum, air, and liquid environments. The AFM has brought about significant progress in a broad range of scientific disciplines and industries such as life sciences [3]-[5], semiconductor manufacturing [6], [7], nanometrology [8], [9], nanofabrication [10], [11] and high-density data storage systems [12], [13].

Fig. 1 illustrates the main components of an AFM. The AFM is a mechanical microscope which uses an atomically sharp tip located at the end of a microcantilever to "feel" the surface of a sample. The tip-sample interaction force causes the microcantilever to deflect and this deflection is measured by a laser and a

Manuscript received June 21, 2013; revised September 9, 2013; accepted October 31, 2013. Date of publication November 25, 2013; date of current version January 6, 2014. This work was supported by Australian Research Council. The review of this paper was arranged by Associate Editor J. T. W. Yeow.

The authors are with the School of Electrical Engineering and Computer Science, The University of Newcastle, Callaghan, NSW 2308, Australia (email: yuenkuan.yong@newcastle.edu.au; ali.bazaei@newcastle.edu.au; reza. moheimani@newcastle.edu.au).

Color versions of one or more of the figures in this paper are available online at http://ieeexplore.ieee.org.

Digital Object Identifier 10.1109/TNANO.2013.2292610

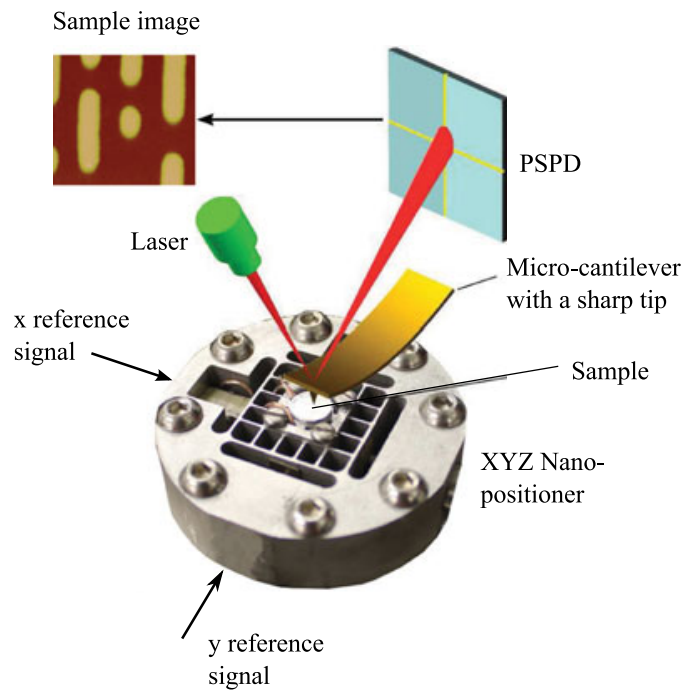

Fig. 1. Basic components of AFM.

position sensitive photodiode (PSPD). A nanopositioner is used to either move the tip or the sample in the $x, y$, and $z$ directions during scanning.

Raster-based scanning has been the standard method of generating images in various forms of scanning probe microscopy, including the STM [14], the scanning near-field optical microscopy [15], scanning capacitance microscopy [16], and the magnetic force microscopy [17]. To construct a raster trajectory, one lateral axis of the nanopositioner is required to track a fast triangular signal, while the other is set to track a slow ramp or staircase signal. The spectrum of the triangle waveform not only consists of its fundamental frequency, but also contains all of its odd harmonics. The nanopositioner has a finite mechanical bandwidth and a highly resonant nature. Tracking of the fast triangle waveform and its harmonics tends to excite the resonance frequency of the nanopositioner [18], [19]. A typical solution to avoid exciting the resonant mode is to limit the fundamental frequency of the triangle waveform to the range 1/100th to 1/10th of the first lateral resonance frequency of the nanopositioner [20]. This clearly impedes the scan rate of AFMs.

A common approach to avoid scan-induced vibrations during video-rate imaging is to use a high-bandwidth flexurebased nanopositioner, with the first lateral resonant peak above $20 \mathrm{kHz}$ [21], [22]. However, designing such a high-bandwidth nanopositioner could be a difficult task. It involves careful considerations of mechanical properties of flexures, as well as electrical properties of piezoelectric stack actuators and drives, preloading of the piezoelectric stack actuators, and manufacturing techniques [23]. In addition, the travel range of a positioner is generally reduced as its resonance frequency is increased [23], [24]. Feedback control methods such as PPF [25], 
resonant control [26], integral resonant control (IRC) [27]-[30], and shunt damping [31], [32] have also been used to suppress resonance frequencies of nanopositioners, and hence, to minimize structural vibrations induced by the raster signal. Another approach to avoid the excitation of structural resonant modes is input-shaping [33], [34]. Feedforward control techniques [19], [24], [35] have also been implemented to improve the tracking performance of nanopositioners during high-speed rastering.

Recently, nonraster scan methods such as spiral-scan [36], cycloid-scan [37], and Lissajous-scan methods [38], [39] have been proposed to significantly increase the scan speed of AFMs. All these nonraster scan methods involve tracking sinusoidal references on the lateral axes of the nanopositioner. Unlike the triangular signals, sinusoids have narrow frequency spectrums. For a nanopositioner with a limited bandwidth, tracking of sinusoidal references is a much easier task than tracking of triangular signals. An example can be found in [38] where vibration-free AFM images were obtained by tracking a $600-\mathrm{Hz}$ Lissajous trajectory in closed-loop using a piezoelectric tube scanner with $825-\mathrm{Hz}$ resonance frequency. These scan results would be difficult to achieve using the conventional raster-scan method.

This paper extends our previous work on high-speed AFM based on tracking Lissajous patterns, reported in [38], to video-rate AFM using a high-speed nanopositioner with lateral resonance frequency of $11.3 \mathrm{kHz}$. An analog integral resonant controller (IRC) is designed and implemented to suppress the first dominant mode of each lateral axis of the nanopositioner. Internal model (IM) controllers are designed for the damped system and implemented to track $1-$ and $2-\mathrm{kHz}$ sinusoidal references. The inclusion of high harmonic components in the IM controllers significantly improves the tracking errors. Compared to the previous video-rate AFM methods reported in [40]-[42] that are based on conventional raster scanning, in open loop, and on higher bandwidth nanopositioners, the proposed video-rate Lissajous scan method uses noise resilient IM controllers and a lower bandwidth nanopositioner. Compared to the low-speed Lissajous scan method presented in [39], which uses linear $H_{\infty}$ controllers, the high-speed method proposed here uses IM control that can compensate for the residual tracking errors due to piezoelectric actuator nonlinear effects such as hysteresis and creep.

The remainder of the paper continues as follows. A brief description of the Lissajous pattern is presented in Section II. Section III outlines the procedures involved in constructing a video-rate Lissajous pattern for the AFM. Section IV discusses the experimental setup. Design and implementation of the ana$\log$ IRC and the IM controllers are discussed in Section V. This section also presents the noise analysis of the open- and closed-loop systems. The open- and closed-loop AFM scans are presented in Section VI. Section VII concludes the paper.

\section{LISSAJOUS PATTERN}

The Lissajous trajectory can be generated by driving the $x$ and $y$-axes of the nanopositioner with purely sinusoidal signals that contain slightly different frequencies, that is,

$$
x(t)=A_{x} \sin \left(2 \pi f_{x} t\right)
$$

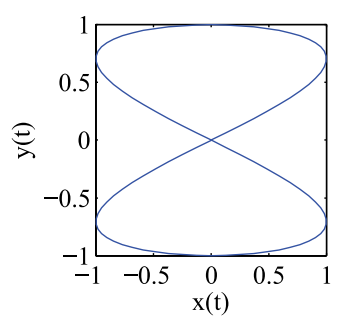

(a)

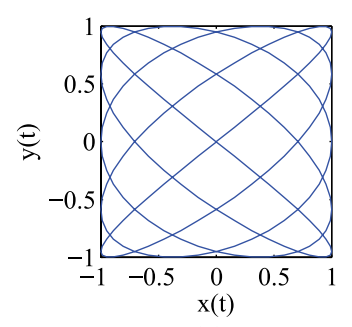

(c)

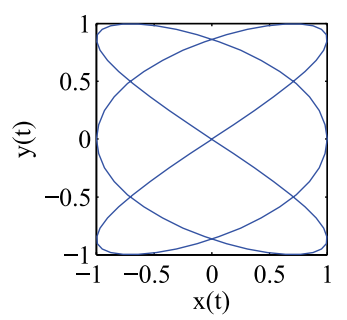

(b)

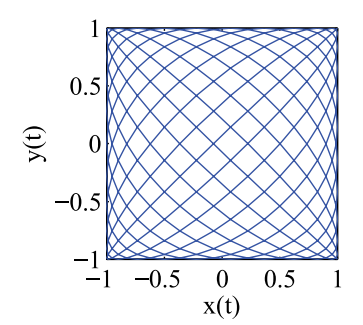

(d)
Fig. 2. Examples of Lissajous patterns with different $f_{x} / f_{y}$ ratio. (a) $f_{x} / f_{y}=2$. (b) $f_{x} / f_{y}=3 / 2$. (c) $f_{x} / f_{y}=5 / 4$. (d) $f_{x} / f_{y}=1.0476$.

$$
y(t)=A_{y} \sin \left(2 \pi f_{y} t\right)
$$

Here, $A_{x}, A_{y}, f_{x}$, and $f_{y}$ are the amplitudes and frequencies of the sinusoids along the $x$ - and $y$-axes, respectively. The shape of the Lissajous pattern is dependent on the ratio $f_{x} / f_{y}$ and the phase difference between the two sinusoids. For simplicity, we assume that the phase difference between the $x$ - and $y$-signals is zero. However, a phase difference can be introduced to generate a variety of Lissajous patterns. Examples of the Lissajous patterns are shown in Fig. 2 for different $f_{x} / f_{y}$ values. Note that the frequency difference between $f_{x}$ and $f_{y}$ also determines the period $T$ in which the pattern evolves and repeats itself [38]. $T$ can be calculated as $T=1 /\left|f_{x}-f_{y}\right|$.

In [38], we reported a detailed analytical framework for generating a Lissajous pattern suitable for AFM. Four examples of this pattern are plotted in Fig. 2(a)-(d). Readers are referred to [38] for a detailed analysis.

\section{Construction of Lissajous Trajectories FOR VIDEO-RATE AFM}

To determine the parameters of the video-rate Lissajous trajectory, we follow the design steps proposed in [38]. These are summarized below.

Step 1: Select the scan size and resolution. A $3 \mu \mathrm{m} \times 3 \mu \mathrm{m}$ area of a sample with a resolution of $h=30 \mathrm{~nm}$ is selected ( $h$ is the largest distance between two scan lines). The intention is to generate an image that is comparable to a raster-scanned image with a resolution of $100 \times 100$ pixels.

Step 2: Calculate $N$. The integer $N$ is calculated by rounding the following equation to the nearest integer:

$$
N=\frac{\pi A_{x} A_{y}}{h \sqrt{A_{x}^{2}+A_{y}^{2}}}
$$

where $A_{x}=A_{y}=1.5 \mu \mathrm{m} . N$ is calculated to be 111 . 
TABLE I

LisSAJOUS PARAMETERS, FRAME RATES, AND SAMPLING FREQUENCIES FOR A SCAN AREA OF $3 \mu \mathrm{M} \times 3 \mu \mathrm{M}$, AND A RESOLUTION OF $h=30 \mathrm{NM}$

\begin{tabular}{cccc}
\hline $\begin{array}{c}f_{x} \\
(\mathrm{~Hz})\end{array}$ & $\begin{array}{c}f_{y} \\
(\mathrm{~Hz})\end{array}$ & $\begin{array}{c}\text { Sampling rate } \\
(\mathrm{Hz})\end{array}$ & $\begin{array}{c}\text { frame rate } \\
(\text { frames/s) }\end{array}$ \\
\hline 1000 & 995.49 & 442000 & 9 \\
2000 & 1990.99 & 884000 & 18 \\
\hline
\end{tabular}

Step 3: Determine the lateral scan frequencies $f_{x}$ and $f_{y} \cdot f_{x}$ represents the scan frequency of the $x$-axis and must fit within the mechanical bandwidth of the nanopositioner; $f_{y}$ can be calculated from

$$
\frac{f_{x}}{f_{y}}=\frac{2 N}{2 N-1} .
$$

Equation (3) ensures that the ratio $f_{x} / f_{y}$ is a rational number. This condition guarantees that the Lissajous trajectory returns to its starting point and repeats itself with the same pattern after one period. We have chosen to perform video-rate AFM at two scan speeds of $f_{x}=1$ and $2 \mathrm{kHz}$. From (3), the respective $f_{y}$ values are 995.49 and $1990.99 \mathrm{~Hz}$.

Step 4: Calculate the frame rate. The frame rate can be calculated by $f_{x} / N$. For $f_{x}=1 \mathrm{kHz}$ and $f_{x}=2 \mathrm{kHz}$, the frame rates are 9 and 18 frames/s, respectively.

Step 5: Calculate the sampling frequency. The sampling frequency is required for data acquisition purposes during AFM imaging. The sampling frequency $f_{s}$ can be obtained from

$$
f_{s}=2(2 N-1) f_{x} .
$$

Table I summarizes the parameters of the two Lissajous trajectories, frame rates, and the corresponding sampling frequencies.

\section{EXPERIMENTAL SETUP}

A high-speed $X Y Z$ flexure-guided nanopositioner, described in [28] and [43] and pictured in Fig. 3, is used to implement the video-rate Lissajous scans in a commercial AFM. The $x$ - and $y$-axes of the nanopositioner are actuated by $5 \mathrm{~mm} \times 5 \mathrm{~mm} \times$ $10 \mathrm{~mm}$ piezoelectric stack actuators. The $z$-axis is moved by a $3 \mathrm{~mm} \times 3 \mathrm{~mm} \times 4 \mathrm{~mm}$ stack actuator. The $x$ - and $y$-actuators are driven by two high-bandwidth voltage amplifiers capable of producing a peak current of $1 \mathrm{~A}$. The $z$-actuator is driven by a PiezoDrive PDL200 voltage amplifier. All voltage amplifiers have a gain of 20 . The resonance frequencies of the two lateral axes are approximately $11.3 \mathrm{kHz}$ and the $z$ resonance frequency is at $65 \mathrm{kHz}$. The lateral and vertical full-range displacements of the nanopositioner are 7 and $3 \mu \mathrm{m}$, respectively. Two 100-kHz bandwidth MicroSense 6810 capacitive sensors are used to measure the $x$ - and $y$-displacements of the nanopositioner. The sensitivity of the sensors is $2.5 \mu \mathrm{m} / \mathrm{V}$. All frequency responses are recorded using a HP35670A dual-channel spectrum analyzer.

Control system implemented for each axis consists of two loops: an inner damping loop which is realized by an analog controller, and an outer tracking loop that implements an IM

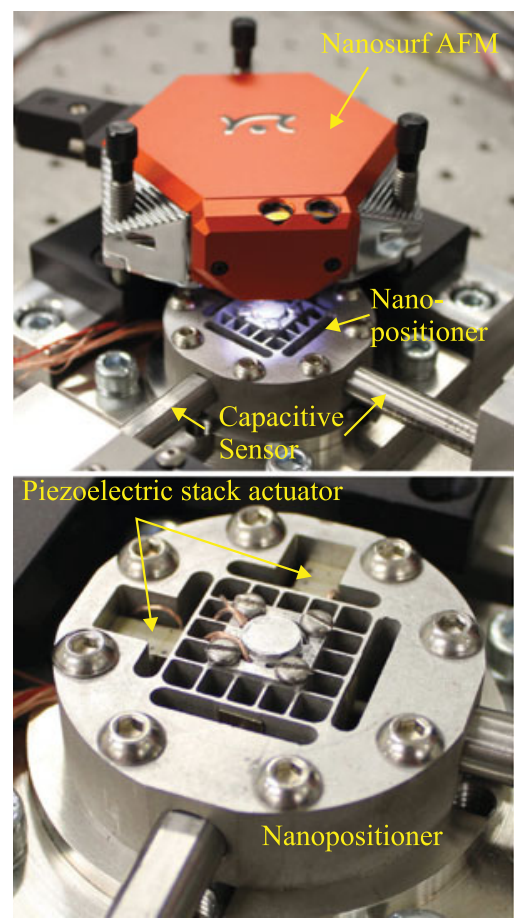

Fig. 3. AFM and a high-speed XYZ nanopositioner.

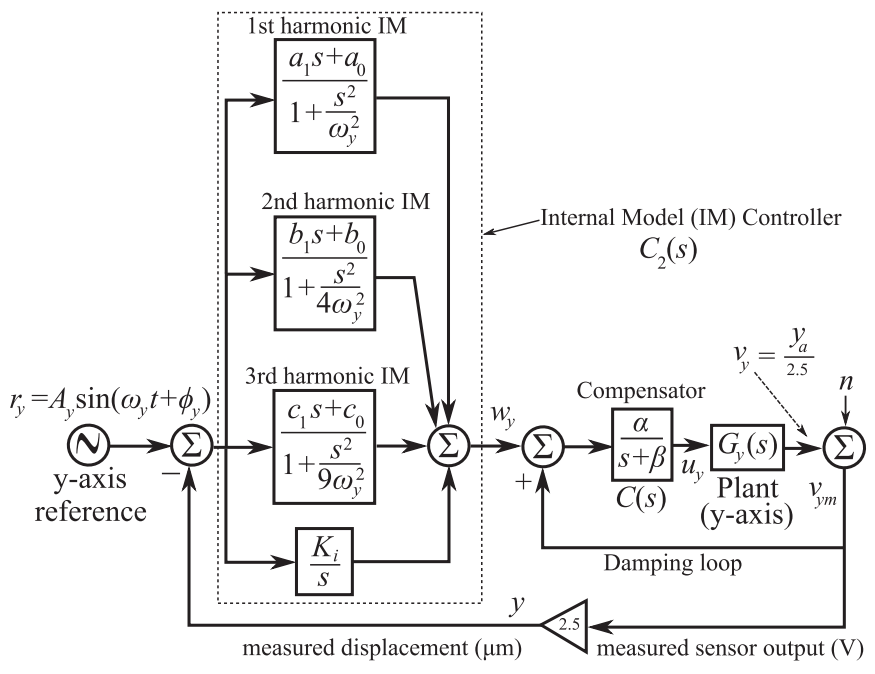

Fig. 4. Block diagram of control system for one axis of scanner.

controller. A dSPACE-1103 rapid prototyping system is used to implement the IM controllers.

\section{Control Design}

Fig. 4 shows the schematic diagram of the control system for the $y$-axis of the stage. Here, the plant, $G_{y}(s)$ refers to the transfer function from the input voltage $u_{y}$ of the high-voltage amplifier (whose output is applied to the $y$-axis piezoelectric actuator) to the voltage $v_{y m}$, measured by the $y$-axis displacement sensor. A similar control system, not shown for brevity, is designed for the $x$-axis.

Open-loop frequency response of the plant is shown in Fig. 5. The following second-order transfer function was fitted to the 
TABLE II

Parameter Values of the Control Systems

\begin{tabular}{cccccccccc}
\hline Reference freq. & $\alpha$ & $\beta$ & $a_{1}$ & $a_{0}$ & $b_{1}$ & $b_{0}$ & $c_{1}$ & $c_{0}$ & $K_{i}$ \\
$1 \mathrm{kHz}$ & $10^{5}$ & $45 \mathrm{k}$ & 0.00024 & 0.24 & 0 & 0 & $2 \times 10^{-6}$ & -0.032 & 800 \\
$2 \mathrm{kHz}$ & $10^{5}$ & $45 \mathrm{k}$ & $2.64 \times 10^{-5}$ & -0.16 & $9.2 \times 10^{-7}$ & -0.014 & 0 & -0.06 & 800 \\
\hline
\end{tabular}
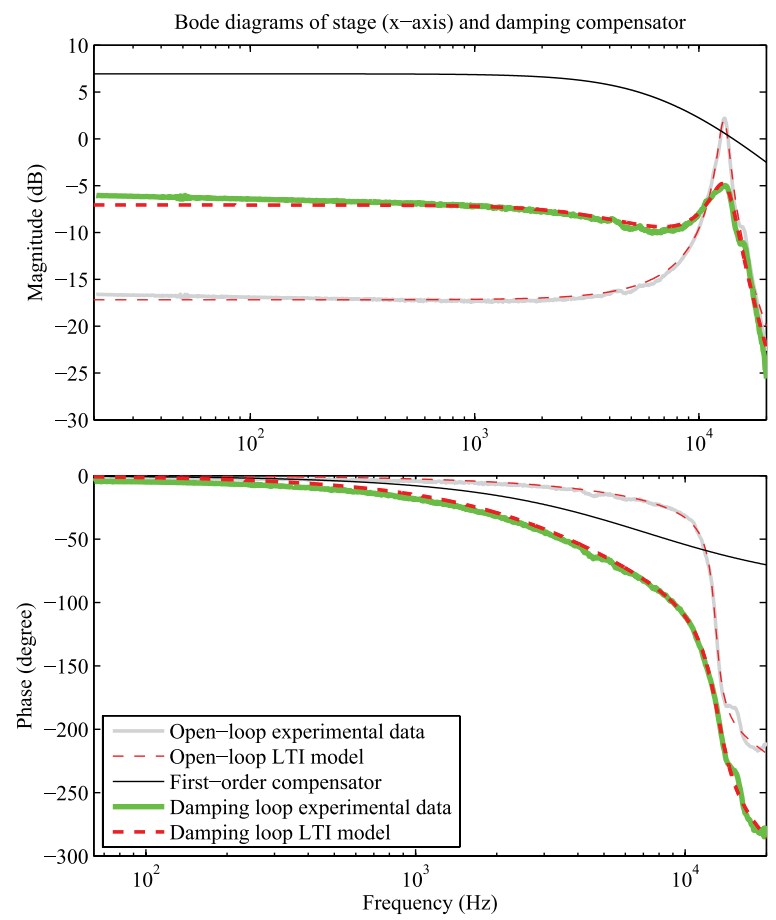

Fig. 5. Frequency responses of the open-loop plant, the compensator, and the damping loop.

experimentally obtained data. Fig. 5 illustrates that this model fits the measured data very closely

$$
G_{y}(s)=\frac{0.01743 s^{2}-5300 s+9.168 \times 10^{8}}{s^{2}+8942 s+6.635 \times 10^{9}} .
$$

The control system for each axis consists of two feedback loops; an inner loop and an outer loop. The inner loop consists of a first-order IRC, denoted by $C(s)$, that is designed to alleviate the highly resonant dynamics of the stage. Parameter values of this compensator are reported in Table II. The associated frequency response is shown in Fig. 5.

The effectiveness of the damping controller can be observed from the experimentally obtained closed-loop frequency response of the inner loop (from the input $w_{y}$ to the sensor output $v_{y m}$ in Fig. 4), plotted in Fig. 5. The IRC reduces the resonance peak from 19 to only $2 \mathrm{~dB}$, which is a significant improvement. The associated stability margins are $10 \mathrm{~dB}$ and $-128^{\circ}$. Given the simple structure of the damping controller, the large bandwidth of the highly resonant plant and sampling rate limitations of our real-time control system (dSPACE-DS1103), we decided to implement the damping controller using the analog circuit shown in Fig. 6. The circuit has a negative de gain which is compensated by out of phase sensing and actuation configurations in the stage (not shown for brevity). The outer loop is digitally implemented using dSPACE.

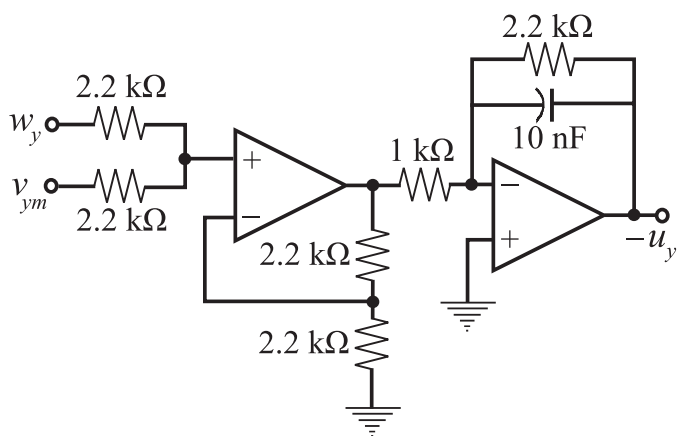

Fig. 6. Circuit diagram of the analog system used to implement the damping loop. The minus sign at the output is for consistency with Fig. 4, where the negative dc gain of the circuit is cancelled by the out-of-phase sensor/actuator configurations of the stage, which is excluded from the plant model (5) for brevity.

Due to the limited sampling rate of $80 \mathrm{kHz}$ in dSPACE, there exist small discontinuities in the discretized controller output $w_{y}$ that cannot excite any vibrations as the inner loop is a welldamped continuous system. If both loops were implemented by dSPACE, such discontinuities would directly be applied to the plant input $u_{y}$, exciting the resonant mode of the stage, leading to undesirable vibrations. Fig. 5 also shows the simulated frequency response of the damping loop using the plant model (5) and the first order compensator model, where a good match with the experimental closed-loop data of the inner loop confirms a valid implementation of the damping loop by the analog circuit. The damping performance is also confirmed by the step responses shown in Fig. 7.

Having well-damped and hence better conditioned plants associated with both $x$ - and $y$-axes, we can incorporate IM controllers in the outer loops to achieve accurate tracking of the sinusoidal set-points as required in Lissajous scan AFM. As shown in Fig. 4, the IM controller $C_{2}(s)$ contains an integrator and a second-order transfer function with imaginary poles that match the fundamental frequency of the sinusoidal reference (first harmonic IM). We have also included second-order transfer functions that correspond to the second and third harmonics of the reference in the controller. These additional controllers can improve the steady-state tracking error resulting from plant nonlinearities such as hysteresis and creep, that manifest as harmonics of the reference frequency [38]. Note that due to the nonlinearities, a pure single-tone sinusoidal actuation is not enough to generate the desired sine wave output. Assuming a static nonlinearity, the ideal actuation waveform generating the desired one-tone output (reference) through a nonlinear system is generally a periodic signal with the same period, which has infinite number of harmonics in addition to its fundamental component. If only one sinusoidal oscillator, at the fundamental frequency, is implemented in the IM controller, it cannot 
(a)

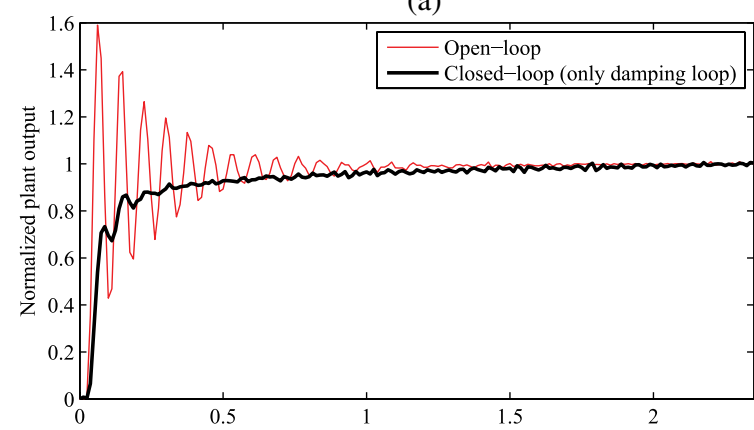

(b)

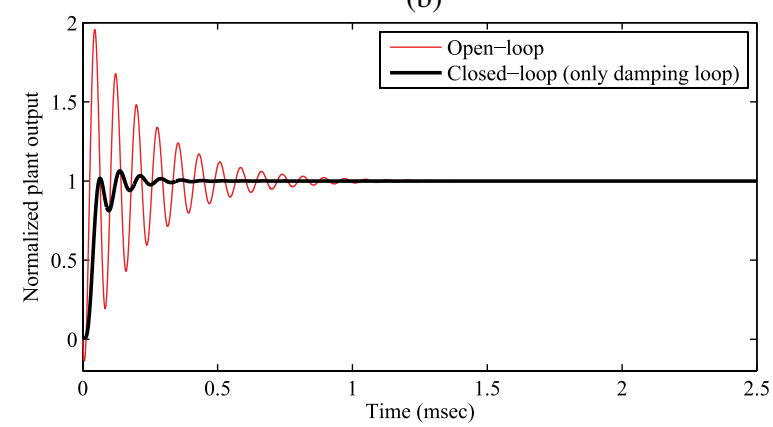

Fig. 7. Normalized step responses of the plant and the damping loop obtained by simulation and experiment. (a) Experimental step responses. (b) Step responses by simulation.

generate the higher harmonics required for the ideal actuation, resulting in a nonzero residual output error.

For the two reference frequencies of 1 and $2 \mathrm{kHz}$, the parameter values of the IM controllers are recorded in Table II and the associated stability margins are $\left(6.8 \mathrm{~dB}, 52^{\circ}\right)$ and $\left(10.8 \mathrm{~dB}, 57^{\circ}\right)$, respectively. Fig. 8 shows the experimentally obtained and the simulated unit-step-responses of the control systems, demonstrating the modeling accuracy and acceptable transient performances in a worst-case scenario.

We are now in a position to experimentally assess the performance of the control system in tracking 1 - and $2-\mathrm{kHz}$ sinusoidal references, required for video-rate Lissajous scans. We also investigate the effectiveness of controlling higher order harmonics in the IM controller. Both $x$ - and $y$-axes of the scanner are simultaneously driven, via their control systems, by sinusoidal references that slightly differ in frequency (by approximately $0.45 \%$ ). Each reference signal has an amplitude of $1.5 \mu \mathrm{m}$ and a zero bias.

Fig. 9 shows the resulting steady-state tracking errors, with and without the third harmonic IM, for the 1-kHz control system. The root-mean-square (RMS) value of the positioning errors is also indicated. It can be observed that incorporating the third harmonic IM in the controller significantly improves the tracking performance. The experimentally obtained results for the 2-kHz control system, shown in Fig. 10, also demonstrate similar improvements, confirming the advantages of the high-order harmonic elements in the IM controller. A nanometer-scale positioning accuracy is achieved while tracking a 2-kHz sinusoidal reference that swings in the micrometer range, by proper control of a nanopositioner whose resonance is at $11.3 \mathrm{kHz}$ and is (a)

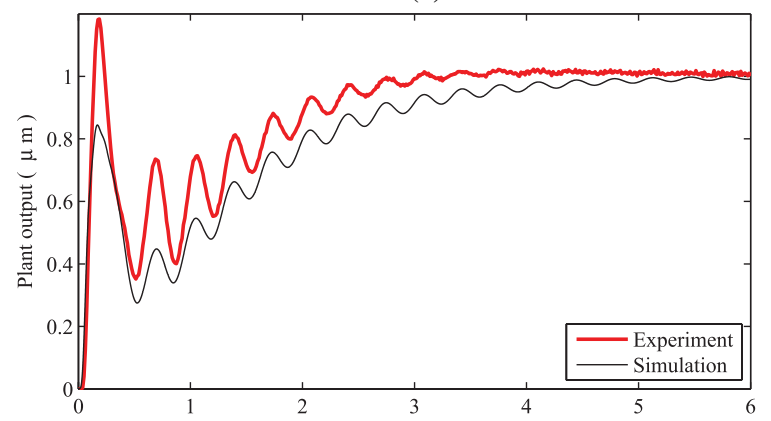

(b)

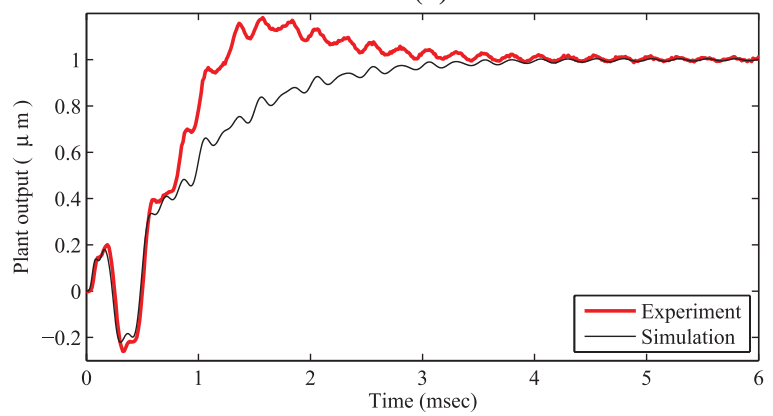

Fig. 8. Unit step responses of the control systems obtained by simulation and experiment. (a) Unit-step-response of closed-loop system with 1-kHz IM controller. (b) Unit-step-response of closed-loop system with 2-kHz IM controller.

(a)

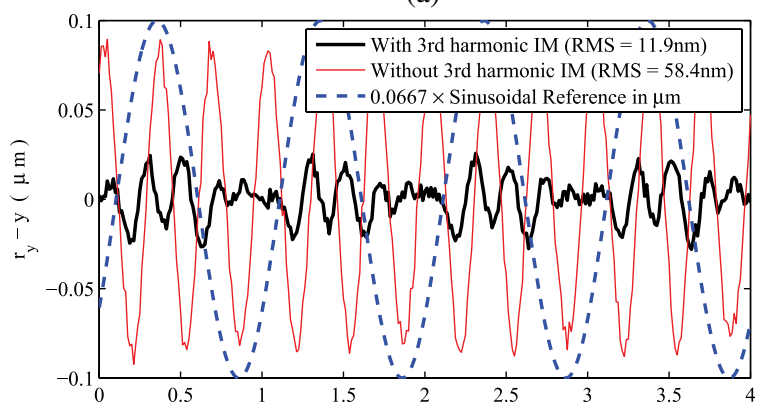

(b)

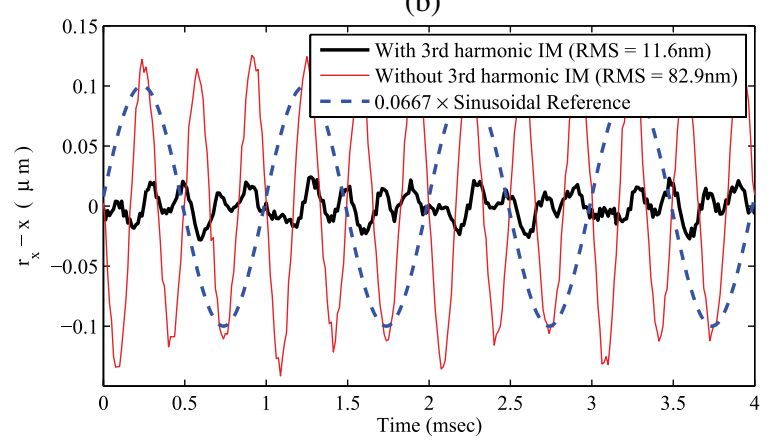

Fig. 9. Experimental steady-state tracking errors of the 1-kHz control system for sinusoidal references with peak-to-peak amplitude of $3 \mu \mathrm{m}$, with and without the third harmonic IM component in controller $C_{2}(s)$. (a) Steady-state tracking errors for $1-\mathrm{kHz}$ control system for $y$-axis. (b) Steady-state tracking errors for 1-kHz control system for $x$-axis.

subjected to uncertainties and nonlinearities such as hysteresis, creep, and cross coupling. 
(a)

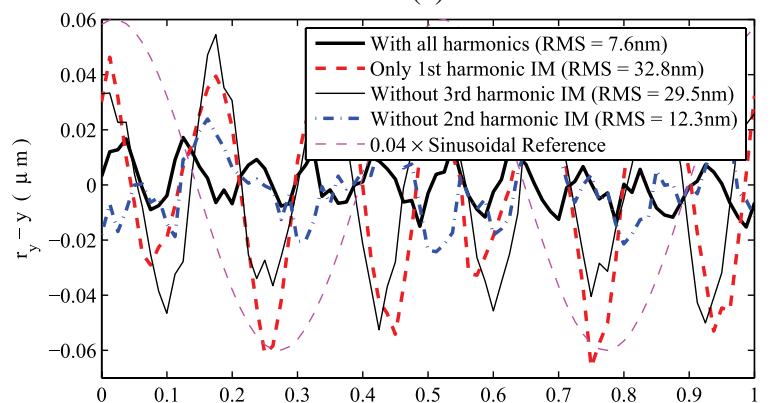

(b)

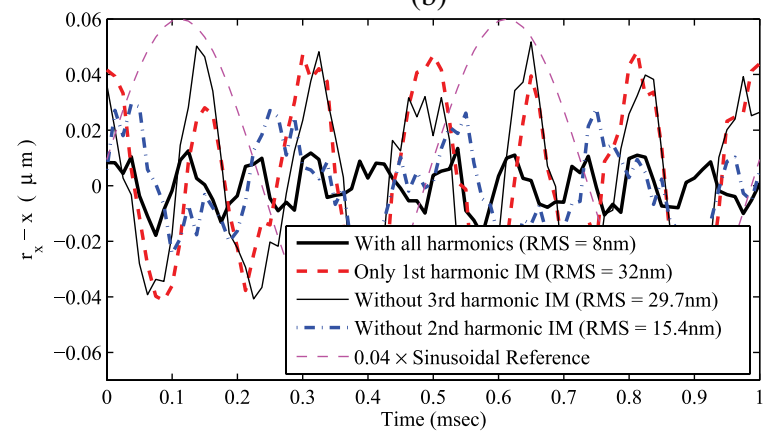

Fig. 10. Experimental steady-state tracking errors of the 2-kHz control system for sinusoidal references with peak-to-peak amplitude of $3 \mu \mathrm{m}$, with and without the third and the second harmonic IM components in controller $C_{2}(s)$. (a) Steady-state tracking errors for $2-\mathrm{kHz}$ control system of $y$-axis. (b) Steadystate tracking errors for $2-\mathrm{kHz}$ control system of $x$-axis.

We are now in a position that we may assess the noise rejection properties of the 1- and 2-kHz control systems. We first measured the sensor noise output of the open-loop system (when the system was not actuated). Using the block diagram of the control system in Fig. 4, the transfer function from the noise source to the actual plant output $y_{a}:=2.5 v_{y}$ (projected noise) is

$$
T_{n}(s):=\frac{y_{a}(s)}{n(s)}=-2.5 \frac{L_{n}(s)}{1+L_{n}(s)}
$$

where $L_{n}=\left(2.5 C_{2}-1\right) C G$, and factor of $2.5 \mu \mathrm{m} / V$ converts the sensor output voltage to displacement. Applying no references, the actual plant output was calculated as the projected noise using the aforementioned plant model. Fig. 11 shows the results in time and frequency domains. Compared to $7.5 \mathrm{~nm} \mathrm{rms}$ value of the noise source, the rms values of the projected noise in 1- and 2-kHz control systems are 1.2 and $1.3 \mathrm{~nm}$, respectively. Hence, the proposed controllers also reduce the effect of sensor noise on the controlled position while maintaining highly acceptable tracking performances.

\section{AFM RESULTS}

This section evaluates the AFM imaging performance of the nanopositioner with the implementation of the proposed control strategies discussed in Section V. The feedback controlled nanopositioner is mounted under a commercial Nanosurf EasyScan 2 AFM, as shown in Fig. 3. Thus, the scanning stage of this commercial AFM is replaced with the high-speed nanopositioner. An ASM 750-HD calibration grating is used to evaluate
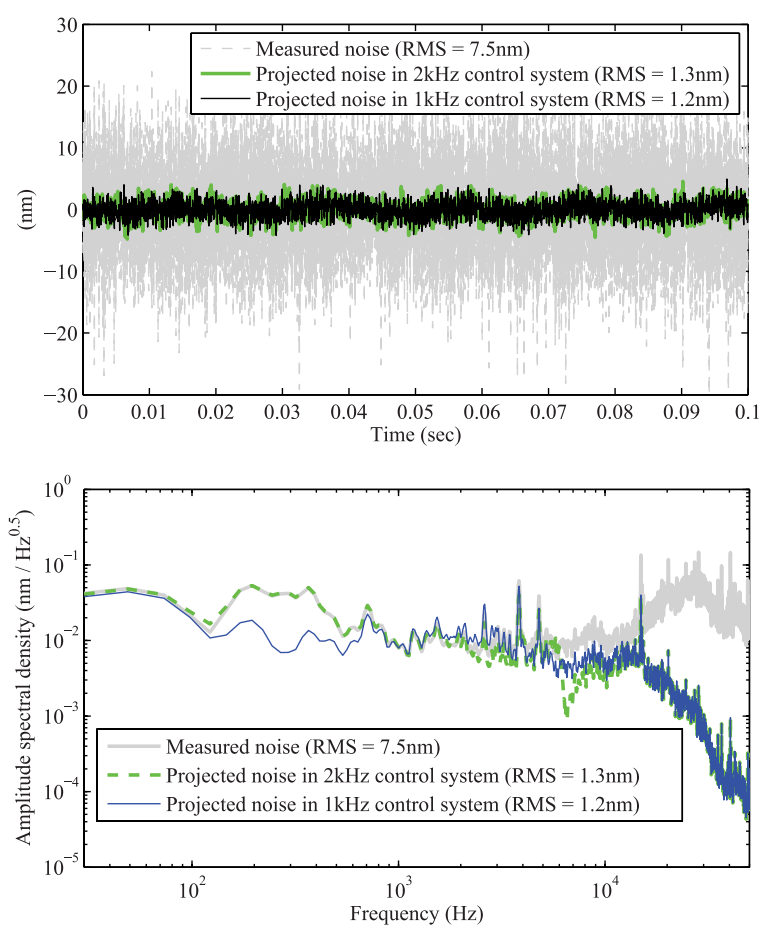

Fig. 11. Measured noise source and the resulting projected noises in the control systems in time and frequency domains.

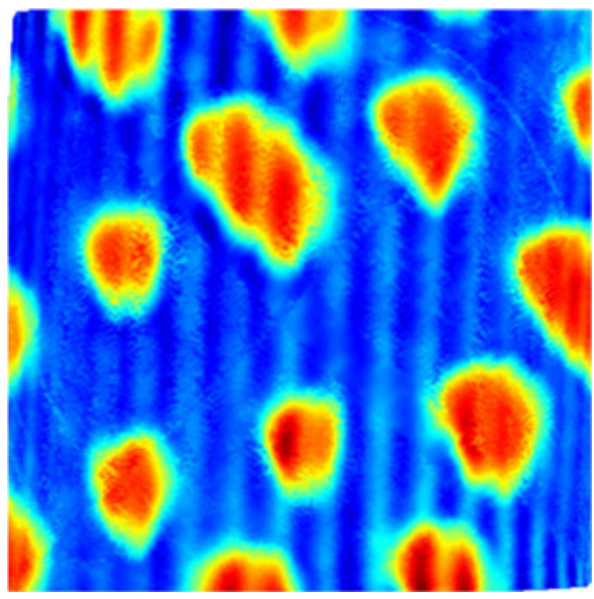

Fig. 12. Open-loop images obtained at 18 frames $/ \mathrm{s}\left(f_{x}=2 \mathrm{kHz}\right)$. The image was severely distorted due to vibrations and poor tracking performance.

the video-rate images. The grating has a step height of $100 \mathrm{~nm}$ and a pitch of $738 \mathrm{~nm}$. A National Instruments $4 \mathrm{MS} / \mathrm{s}$ PXI-6124 data acquisition card is used to record the images.

AFMs can be operated in contact, semicontact, and dynamic modes. Details of these scanning modes can be found in [44], [19] and [23]. AFM images presented here were obtained in constant-height contact-mode. In this mode, the vertical feedback loop is deactivated during scanning. The 3-D topographic images of the sample were constructed by plotting the deflection of the microcantilever versus the $x$ - and $y$-positions of the sample.

Fig. 12 shows the open-loop image of a $3 \mu \mathrm{m} \times 3 \mu \mathrm{m}$ sample area obtained at 18 frames $/ \mathrm{s}\left(f_{x}=2 \mathrm{kHz}\right)$. The image was 

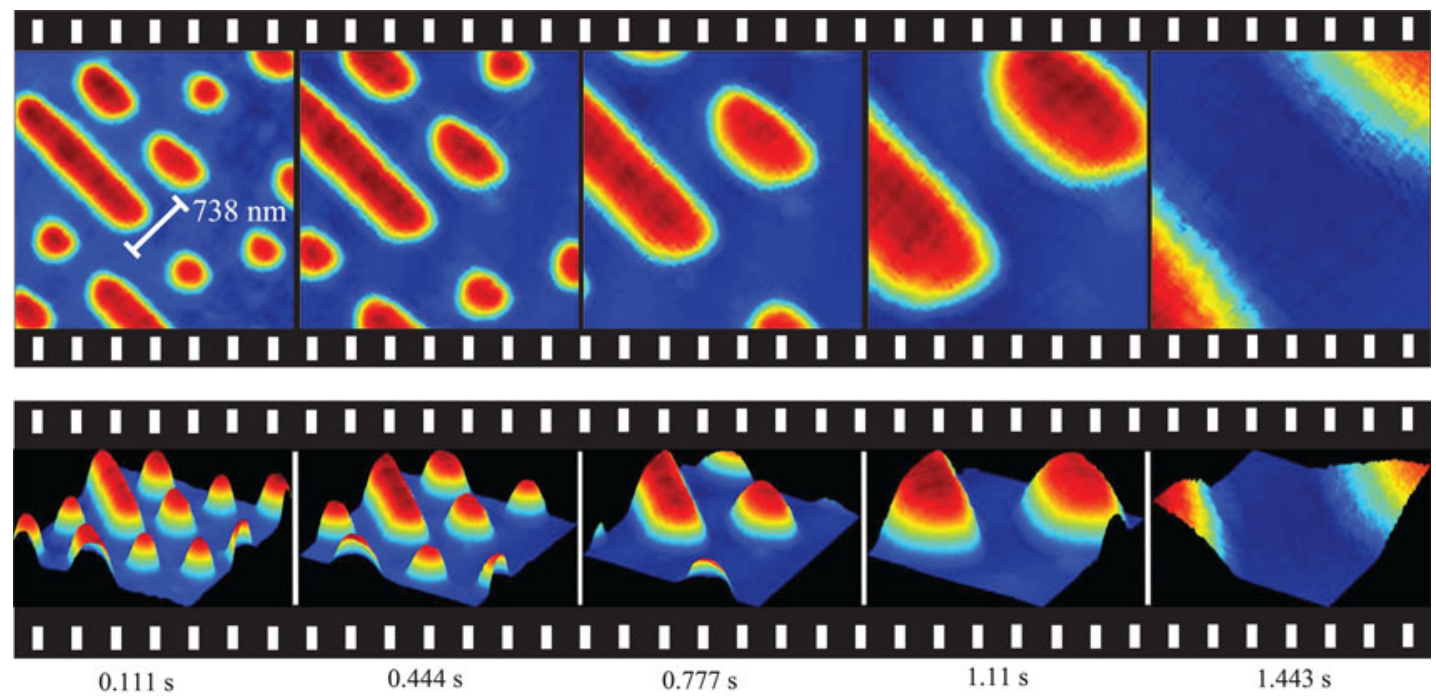

(a)
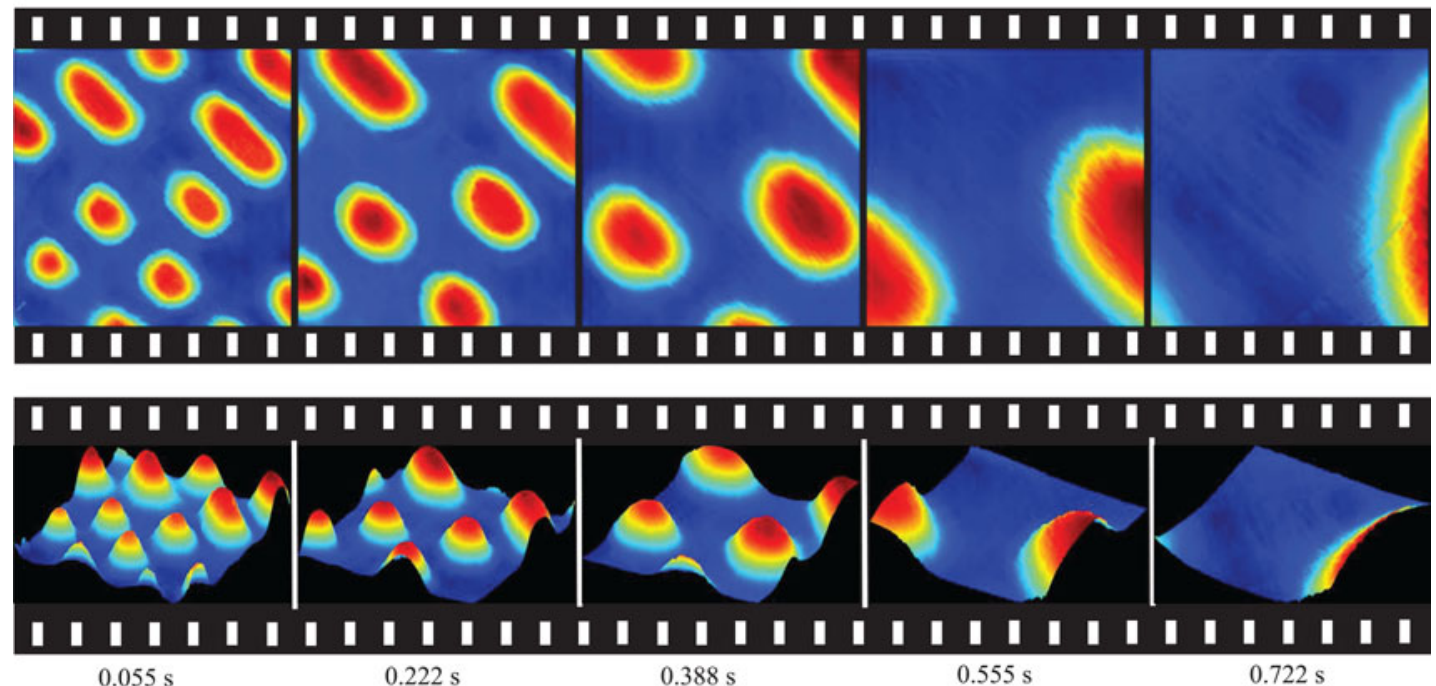

(b)

Fig. 13. Sequence of Lissajous images showing the process of fast zooming in of a sample area from $3 \mu \mathrm{m} \times 3 \mu \mathrm{m}$ to $1 \mu \mathrm{m} \times 1 \mu \mathrm{m}$. Every third image in the series is illustrated above. These series of images were captured at (a) 9 frames $/ \mathrm{s}\left(f_{x}=1 \mathrm{kHz}\right)$ and (b) $18 \mathrm{frames} / \mathrm{s}\left(f_{x}=2 \mathrm{kHz}\right)$.

severely distorted due to vibrations and poor tracking performance of the nanopositioner in open loop.

Fig. 13 illustrates a sequence of closed-loop Lissajous images which were obtained at 9 frames/s $\left(f_{x}=1 \mathrm{kHz}\right)$ and 18 frames/s $\left(f_{x}=2 \mathrm{kHz}\right.$ ). The images show a rapid zooming-in process on a sample area from $3 \mu \mathrm{m} \times 3 \mu \mathrm{m}$ to $1 \mu \mathrm{m} \times 1 \mu \mathrm{m}$. Every third image in the sequence is shown in the figure. With the proposed control strategies implemented, the image artifacts due to vibrations and poor tracking were eliminated.

\section{CONCLUSION}

In this paper, a nonraster scan method based on following a Lissajous pattern was used to achieve video-rate atomic force microscopy on a $11.3-\mathrm{kHz}$ nanopositioner. An analog integral resonant controller was designed and implemented to suppress the first resonant mode of each lateral axis of the nanopositioner by $17 \mathrm{~dB}$. IM controllers incorporating higher harmonic components were used to significantly improve the tracking errors of the scanner. It was shown that the projected noise is much smaller than the sensor noise. This implies that the proposed control strategies are capable of reducing the effect of sensor noise at the controlled position and simultaneously achieving high tracking performances. By combining the Lissajous-scan method with the proposed IRC and IM controllers, high-quality AFM images were successfully recorded at 9 and 18 frames/s.

\section{REFERENCES}

[1] G. Binnig, H. Rohrer, C. Gerber, and E. Weibel, "Surface studies by scanning tunneling microscopy," vol. 49, no. 1, pp. 57-61, 1982.

[2] G. Binnig, C. F. Quate, and C. Gerber, "Atomic force microscope," vol. 56, pp. 930-933, 1986.

[3] T. Ando, "High-speed atomic force microscopy coming of age," Nanotechnology, vol. 23, no. 6, p. 062001, 2012.

[4] T. Ando, T. Uchihashi, N. Kodera, D. Yamamoto, A. Miyagi, M. Taniguchi, and H. Yamashita, "High-speed AFM and nano-visualization of biomolecular processes," Pflügers Archiv. Eur. J. Physiol., vol. 456, no. 1, pp. 211-225, 2008. 
[5] P. Hinterdorfer and Y. F. Dufrene, "Detection and localization of single molecular recognition events using atomic force microscopy," Nature Methods, vol. 3, pp. 347-355, 2006.

[6] K. Wiesauer and G. Springholz, "Fabrication of semiconductor nanostructures by nanoindentation of photoresist layers using atomic force microscopy," J. Appl. Phys., vol. 88, no. 12, pp. 7289-7297, 2000.

[7] R. A. Oliver, "Advances in AFM for the electrical characterization of semiconductors," Rep. Progr. Phys., vol. 71, no. 7, p. 076501, 2008.

[8] Y. Hua, C. Coggins, and S. Park, "Advanced 3D metrology atomic force microscope," in Proc. IEEE/SEMI Adv. Semicond. Manuf. Conf., 2010, pp. $7-10$.

[9] L. Tetard, A. Passian, R. H. Farahi, B. H. Davison, S. Jung, A. J. Ragauskas, A. L. Lereu, and T. Thundat, "Nanometrology of delignified Populus using mode synthesizing atomic force microscopy," Nanotechnology, vol. 22, no. 46, p. 465702, 2011.

[10] J. A. Vicary and M. J. Miles, "Real-time nanofabrication with high-speed atomic force microscopy," Nanotechnology, vol. 20, no. 9, p. 095302, 2009.

[11] S. Kim and S.-W. Kim, "AFM-based nanofabrication with assistance of femtosecond pulse laser radiation," J. Phys.: Conf. Series, vol. 61, no. 1, pp. 550-554, 2007.

[12] G. Binnig, M. Despont, U. Drechsler, W. Haberle, M. Lutwyche, P. Vettiger, H. Mamin, B. Chui, and T. Kenny, "Ultrahigh-density atomic force microscopy data storage with erase capability," Appl. Phys. Lett. vol. 74, no. 9, pp. 1329-1331, 1999.

[13] P. Vettiger, G. Cross, M. Despont, U. Drechsler, U. Dürig, B. Gotsmann, W. Häberle, M. A. Lantz, H. E. Rothuizen, R. Stutz, and G. K. Binnig, "The 'Milipede'-Nanotechnology entering data storage," vol. 1, no. 1, pp. 39-55, 2002

[14] G. Binnig and H. Rohrer, "The scanning tunneling microscope," Scientif. Amer., vol. 253, pp. 50-56, 1986.

[15] D. W. Pohl, U. C. Fischer, and U. T. Drig, "Scanning near-field optical microscopy (SNOM)," J. Microsc., vol. 152, no. 3, pp. 853-861, 1988.

[16] C. C. Williams, W. P. Hough, and S. A. Rishton, "Scanning capacitance microscopy on a $25 \mathrm{~nm}$ scale," Appl. Phys. Lett., vol. 55, no. 2, pp. 203 205, 1989.

[17] Y. Martin and H. K. Wickramasinghe, "Magnetic imaging by "force microscopy' with 1000 [A-ring] resolution," Appl.Phys. Lett., vol. 50, no. 20, pp. $1455-1457,1987$

[18] S. O. R. Moheimani, "Invited review article: Accurate and fast nanopositioning with piezoelectric tube scanners: Emerging trends and future challenges," Rev. Scientif. Instrum., vol. 79, no. 7, p. 071101, 2008.

[19] G. M. Clayton, S. Tien, K. K. Leang, Q. Zou, and S. Devasia, "A review of feedforward control approaches in nanopositioning for high-speed SPM," J. Dyn. Syst., Meas., Control, vol. 131, no. 6, p. 061101, 2009.

[20] S. Devasia, E. Eleftheriou, and S. O. R. Moheimani, "A survey of control issues in nanopositioning," vol. 15, pp. 802-823, 2007.

[21] G. Schitter, K. J. Åstrom, B. DeMartini, P. J. Thurner, K. L. Turner, and P. K. Hansma, "Design and modeling of a high-speed AFM-scanner," vol 15, no. 5, pp. 906-915, 2007.

[22] B. J. Kenton and K. K. Leang, "Design and control of a three-axis serialkinematic high-bandwidth nanopositioner," IEEE/ASME Trans. Mechatronics, vol. 17, no. 2, pp. 356-368, 2012

[23] Y. K. Yong, S. O. R. Moheimani, B. J. Kenton, and K. K. Leang, "Invited review article: High-speed flexure-guided nanopositioning: Mechanical design and control issues," Rev. Scientif. Instrum., vol. 83, no. 12, p. 121101,2012

[24] Y. K. Yong, S. Aphale, and S. O. R. Moheimani, "Design, identification and control of a flexure-based XY stage for fast nanoscale positioning," IEEE Trans. Nanotechnol., vol. 8, no. 1, pp. 46-54, Jan. 2009.

[25] B. Bhikkaji, M. Ratnam, A. J. Fleming, and S. O. R. Moheimani, "Highperformance control of piezoelectric tube scanners," vol. 15, no. 5, pp. 853-866, 2007.

[26] A. Sebastian, A. Pantazi, S. O. R. Moheimani, H. Pozidis, and E. Eleftheriou, "Achieving sub-nanometer precision in a MEMS storage device during self-servo write process," IEEE Trans. Nanotechnol., vol. 7, no. 5, pp. 586-595, Sep. 2008.

[27] S. S. Aphale, A. J. Fleming, and S. O. R. Moheimani, "Integral resonant control of collocated smart structures," Smart Mater. Struct., vol. 16, pp. 439-446, 2007.

[28] Y. K. Yong, B. Bhikkaji, and S. O. R. Moheimani, "Design, modeling and FPAA-based control of a high-speed atomic force microscope nanopositioner," IEEE/ASME Trans. Mechatronics, vol. 18, no. 3, pp. 1060-1071, Jun. 2013
[29] A. J. Fleming, S. Aphale, and S. O. R. Moheimani, "A new method for robust damping and tracking control of scanning probe microscope positioning stages," IEEE Trans. Nanotechnol., vol. 9, no. 4, pp. 438-448, Jul. 2010

[30] Y. K. Yong, A. Fleming, and S. O. R. Moheimani, "A novel piezoelectric strain sensor for simultaneous damping and tracking control of a highspeed nanopositioner," IEEE/ASME Trans. Mechatronics, vol. 18, no. 3, pp. 1113-1121, Jun. 2013

[31] S. Aphale, A. J. Fleming, and S. O. R. Moheimani, "High speed nanoscale positioning using a piezoelectric tube actuator with active shunt control," Micro Nano Lett., vol. 2, no. 1, pp. 9-12, 2007.

[32] A. A. Eielsen and A. J. Fleming, "Passive shunt damping of a piezoelectric stack nanopositioner," in Proc. Amer. Control Conf., 2010, pp. 4963-4968.

[33] G. Schitter, P. J. Thurner, and P. K. Hansma, "Design and input-shaping control of a novel scanner for high-speed atomic force microscopy," Mechatronics, vol. 18, pp. 282-288, 2008.

[34] A. J. Fleming and A. G. Wills, "Optimal periodic trajectories for bandlimited systems," IEEE Trans. Control. Syst. Technol., vol. 13, no. 3, pp. 552-562, May 2009.

[35] K. K. Leang and S. Devasia, "Feedback-linearized inverse feedforward for creep, hysteresis, and vibration compensation in piezoactuators," IEEE Control Syst. Technol.: Spec. Issue Dyn. Control Micro-Nano-Scale Syst., vol. 15 , no. 5, pp. 927-935, Sep. 2007.

[36] I. A. Mahmood, S. O. R. Moheimani, and B. Bhikkaji, "A new scanning method for fast atomic force microscopy," IEEE Trans. Nanotechnol., vol. 10, no. 2, pp. 203-216, Mar. 2011

[37] Y. K. Yong, S. O. R. Moheimani, and I. R. Petersen, "High-speed cycloidscan atomic force microscopy," Nanotechnology, vol. 21, no. 36, p. 365503, 2010.

[38] A. Bazaei, Y. K. Yong, and S. O. R. Moheimani, "High-speed Lissajousscan atomic force microscopy: Scan pattern planning and control design issues," Rev. Scientif. Instrum., vol. 83, no. 6, p. 063701, 2012.

[39] T. Tuma, J. Lygeros, V. Kartik, A. Sebastian, and A. Pantazi, "Highspeed multiresolution scanning probe microscopy based on Lissajous scan trajectories," Nanotechnology, vol. 23, no. 18, p. 185501, 2012.

[40] A. D. L. Humphris, J. K. Hobbs, and M. J. Miles, "Ultrahigh-speed scanning near-field optical microscopy capable of over 100 frames per second," Appl. Phys. Lett., vol. 83, pp. 6-8, 2003.

[41] A. D. L. Humphris, M. J. Miles, and J. K. Hobbs, "A mechanical microscope: High-speed atomic force microscopy," Appl. Phys. Lett., vol. 86, p. 034106, 2005

[42] T. Ando, T. Uchihashi, and T. Fukuma, "High-speed atomic force microscopy for nano-visualization of dynamic biomolecular processes," Progr. Surf. Sci., vol. 83, pp. 337-437, 2008.

[43] Y. K. Yong and S. O. R. Moheimani, "Design of an inertially counterbalanced Z-nanopositioner for high-speed atomic force microscopy," IEEE/ASME Trans. Nanotechnol., vol. 12, no. 2, pp. 137-145, Mar. 2013.

[44] D. Abramovitch, S. Andersson, L. Pao, and G. Schitter, "A tutorial on the mechanisms, dynamics, and control of atomic force microscopes," in Proc. Amer. Control Conf., Jul. 2007, pp. 3488-3502.

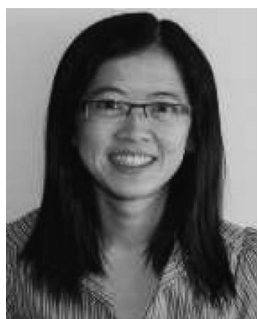

Yuen Kuan Yong (M'09) received the B.Eng. degree (first Class Hons.) in mechatronic engineering and the $\mathrm{Ph} . \mathrm{D}$. degree in mechanical engineering from The University of Adelaide, Adelaide, Australia, in 2001 and 2007, respectively.

She is currently an Australian Research Council DECRA Fellow with the School of Electrical Engineering and Computer Science, The University of Newcastle. Her research interests include the design and control of nanopositioning systems, high-speed atomic force microscopy, finite-element analysis of smart materials and structures, and sensing and actuation.

Dr. Yong was a recipient of the 2008 IEEE/ASME International Conference on Advanced Intelligent Mechatronics (AIM) Best Conference Paper Finalist Award. She is a member of the Technical Program Committee of AIM and the International Conference on Manipulation, Manufacturing, and Measurement on the Nanoscale (3M-NANO). She is also an Associate Editor of the Editorial Board of the International Journal of Advanced Robotic Systems. 


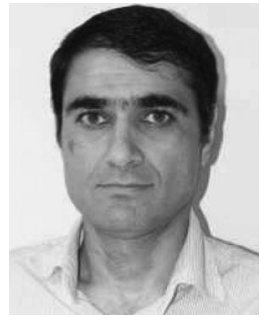

Ali Bazaei (M'01) received the B.Sc. and M.Sc. degrees from Shiraz University, Shiraz, Iran, and the $\mathrm{Ph} . \mathrm{D}$. degrees from University of Western Ontario, London, ON, Canada, and Tarbiat Modares University, Tehran, Iran, in 1992, 1995, 2004, and 2009, respectively, all in electrical engineering.

From September 1995 to January 2000, he was an Instructor with Yazd University, Yazd, Iran. From September 2004 to December 2005, he was a Research Assistant with the Department of Electrical and Computer Engineering, University of Western Ontario, London, ON, Canada. He is currently a research academic with the School of Electrical Engineering and Computer Science, The University of Newcastle, Australia. His research interests include the general area of nonlinear systems including control and modeling of structurally flexible systems, friction modeling and compensation, neural networks, and microposition sensors.

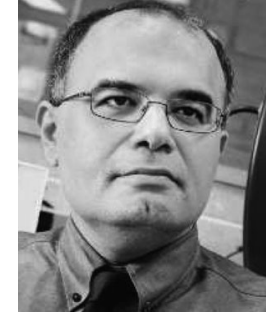

S. O. Reza Moheimani (M'07-F'01) received the B.Sc. degree in electrical and electronic engineering from Shiraz University, Shiraz, Iran, in 1990 and the M.Eng.Sc. and Ph.D. degrees in electrical and electronic engineering from the University of New South Wales, Sydney, New South Wales, Australia, at the Australian Defence Force Academy, Canberra, Australia, in 1993 and 1996, respectively.

He joined The University of Newcastle, Callaghan, Australia, in 1997, where he is a Professor of electrical engineering and an Australian Research Council ARC) Future Fellow. He is the Founder and the Director of the Laboratory for Dynamics and Control of Nanosystems, a multimillion-dollar state-of-the-art research facility. During 2003-2010, he was the Associate Director of the ARC Centre of Excellence for Complex Dynamic Systems and Control. His current research interests include area of ultrahigh-precision mechatronic systems, with particular emphasis on dynamics and control at the nanometer scale, including applications of control and estimation in nanopositioning systems for high-speed scanning probe microscopy, modeling and control of microcantilever-based devices, control of microactuators in microelectromechanical systems, and control issues related to ultrahigh-density probe-based data-storage systems.

Prof. Moheimani is a Fellow of the International Federation of Automatic Control (IFAC) and the Institute of Physics (U.K.). He is a corecipient of the 2007 IEEE TRANSACTIONS ON CONTROL SYSTEMS TECHNOLOGY Outstanding Paper Award and the 2009 IEEE Control Systems Technology Award, together with a group of researchers from IBM Zurich Research Labs, where he has held several visiting appointments. He has served on the editorial boards of a number of journals, including the IEEE/ASME TRANSACTIONS ON MECHATRONICS, IEEE TRANSACTIONS ON CONTROL SYSTEMS TECHNOLOGY, and Control Engineering Practice. He has chaired several international conferences and workshops and currently chairs the IFAC Technical Committee on Mechatronic Systems. 